\title{
Design of a High Torque Density In-Wheel YASA AFPM Motor
}

\author{
A. Di Gerlando, G. Foglia, C. Ricca
}

\begin{abstract}
An analytical design approach of a YASA Permanent magnet in-wheel motor for automotive applications is presented. Making use of the sizing equations, some key design parameters are linked to the heat transfer thermal circuit equations. The procedure allows to retrieve the design quantities which satisfy both the thermal and electromagnetic constraints. Finally, an approach to obtain the winding data and a brief analysis of the overload thermal condition is presented.
\end{abstract}

Index Terms-AFPM, YASA, liquid cooling, automotive, inwheel motor, permanent magnet.

\section{NOMENCLATURE}

$A_{m}, A_{t}, A_{p l}=$ PM flux, stator tooth, copper strip area. $b_{m}, \tau_{m}, \alpha_{m}=$ PM peripheral size, pole pitch, p.u. ratio $\frac{b_{m}}{\tau_{m}}$. $b_{p s}=$ tooth head lateral peripheral extension $\frac{b_{s}-b_{s o}}{2}$. $b_{s}, b_{s o}=$ slot, slot opening peripheral size.

$b_{t e}, b_{t i}$ stator tooth external, internal peripheral size.

$B, B_{1}=$ air gap flux density and its $1^{\text {st }}$ harmonic at no load. $B_{r}, B_{m}, \mu_{r_{p u}}=$ PM residual, PM flux density at no load, relative permeability.

$B_{t}, B_{p s}, B_{d r}=$ tooth, pole shoe, rotor disk flux density at no load.

$c_{w}, \gamma_{w}=$ water specific heat, density.

$d_{c}=$ distance between two adjacent coils inside the slot

$2 \cdot\left(d_{c h}+w_{c h}+w_{\text {pot }}\right)+w_{g}$.

$d_{c h}=$ cooling channel width.

$d_{w}=$ cooling channel hydraulic diameter $\frac{2 \cdot d_{c h}}{1+\frac{d_{c h}}{h_{c o i l}}}$.

$D_{i}, D_{e}, \rho_{D}=$ internal, external diameter, diameter ratio $\frac{D_{i}}{D_{e}}$.

$h_{m}, s_{d r}, g, h_{\text {coil }}=$ PM, rotor disk, air gap, coil height.

$h_{p s}, h_{s o}=$ stator tooth head, slot opening height.

$I_{p h}, M_{c o i l}, S, \Delta=$ phase current, coil M.M.F, superficial and axial linear current density.

$k_{c}, k_{s t}, k_{w}, k_{d}=$ carter, stacking, winding, distribution factor.

$l_{\text {cool }}=$ wet cooling surface perimeter of one coil

$l_{\text {turn }}+4 \cdot\left[w_{\text {coil }}+2 \cdot\left(w_{\text {en }}+w_{\text {pot }}\right)\right]$.

$l_{\text {turn }}=$ average turn length $2 \cdot L+b_{t e}+b_{t i}+4 \cdot w_{\text {coil }}$.

$L_{r}, L=$ rotor disk, stator core radial size.

$N_{c_{p h}}, N_{c}=\mathrm{N}^{\circ}$ of coil/pole/phase, electromagnetic cycles.

$N_{n}, N_{p}, \rho_{N}=$ rated, peak mechanical speed, speed ratio $\frac{N}{N_{n}}$.

$N_{t}, N_{m}, N_{t c}=\mathrm{N}^{\circ}$ of stator teeth, PM per side, coil turns.

$P_{c u}, P_{w}=$ coil joule loss, power dissipated by the cooling surface.

$R_{p h}, X_{p h}, L_{1}=$ phase synchronous resistance, reactance,

A. Di Gerlando, G. Foglia, C. Ricca are with Dpt. of Energy, Politecnico di Milano, via La Masa 34, 20156 Milano - Italy [antonino.digerlando, gianmaria.foglia, claudio.ricca] @ polimi.it. single turn coil inductance.

$R_{t h_{e n}}, R_{t h_{p o t}}, R_{t h_{w}}=$ enamel, potting, water thermal resistance.

$T_{a}, T_{c u}=$ ambient, copper temperature.

$T_{n}, T_{p}, \rho_{T}=$ rated, peak torque, torque ratio $\frac{T}{T_{n}}$.

$T_{w_{\text {in }}}, T_{w_{\text {out }}}, T_{w_{\text {av }}}=$ inlet, outlet, average flowing water temperature.

$v_{w}, Q=$ water average speed, cooling channel water flow.

$V_{c u}, c_{c u}, \gamma_{c u},=$ copper volume, specific heat, density.

$V_{p h}, E_{p h}, \rho_{E V}=$ phase voltage, B.E.M.F, voltage ratio $\frac{E_{p h}}{V_{p h_{p}}}$.

$w_{\text {coil }}=$ copper width $\frac{b_{s}}{2}-\frac{d_{c}}{2}-w_{c h}$.

$w_{e n}, w_{\text {pot }}, w_{c h}=$ enamel, potting, cooling channel thickness.

$w_{g}=$ empty space between two adjacent cooling channels.

$w_{p l}, s_{p l}=$ copper strip width and height.

$\alpha_{t}=$ electrical angle between adjacent coils $\frac{\pi}{N_{m}+1}$.

$\alpha_{w}, K_{t h}=$ convection, global thermal exchange coefficient.

$\theta=$ copper and inlet water temperature difference $T_{c u}-T_{w_{i n}}$.

$\lambda_{\text {en }}, \lambda_{\text {pot }}=$ enamel, potting thermal conductivity.

$\Phi_{g}, \Phi_{1}=$ air gap and its $1^{\text {st }}$ harmonic flux at no load.

$\Phi_{m}, \Phi_{l}, \eta_{M g}=$ PM, leakage flux, air gap efficiency $\frac{\Phi_{g}}{\Phi_{m}}$.

$\rho_{c u}, \alpha_{c u}, \alpha_{T_{c u}}=$ copper resistivity, filling factor, resistivity thermal coefficient.

$\omega_{e}, r, x=$ angular frequency, p.u phase resistance, reactance. ${ }_{n, p}, t_{h}=$ rated, peak, theoretical subscript.

\section{INTRODUCTION}

A lthough internal combustion engine have been dominating the past century, their domain is now slowly coming to an end. Some of the reasons of this gradual substitution is due to their low efficiency and carbon dioxide emissions but as well because of their inferiority regarding noise, control and stall torque.

Inside the electrical mobility industry, permanent magnet machines are still the most used ones and several are the works in the literature showing their advantages like high efficiency, high torque density, absence of excitation currents, low vibration and noise [1].

The following paper describes the steps to obtain analytically an approximate design of an high torque density in-wheel axial motor for automotive applications taking into account the thermal machine limits as well. The geometrical restrictions related to the rims of the wheel, explain the reason to adopt a configuration which shows a predominant radius over the machine core length [2], [3]. To this purpose, starting from the equivalent circuit of the axial machine during no load conditions, the $1^{\text {st }}$ harmonic air gap flux density RMS 
value has been retrieved. By mean of the sizing equations the current density necessary to get the rated torque is calculated.

Due to the fact that the torque density requested is massive and so the current, in order to dissipate the power losses generated by its flow inside the machine coils, a proper cooling system strategy for the machine must be implemented. At the considered power densities the use of air cooling is not enough to dissipate all the heat generated, and for this reason the choice falls on a water cooling solution. By the use of a simplified thermal circuit model, which takes into account the conduction and convection phenomena, the geometrical parameters of the motor can be written as a function of the current density.

Solving the non linear system generated by the sizing and the thermal equations leads to a solution that satisfies them both. After a brief analysis about how to retrieve the winding data of the motor, the overload thermal condition is modeled and analytical results are validated by some 3D FEM simulations.

\section{Design Procedure And Machine Type}

The machine type which has been chosen for this application is a single stator double rotor YASA configuration. Similarly to the procedure carried out in [4], the starting data are specified for a reference operating point, at a certain torque and speed level, together with its thermal specifications.

Precisely, the operating point which has been taken into consideration as the design rated point, is the maximum power value inside the constant torque region, during the most disadvantageous cooling/ambient thermal conditions. Some of the geometrical and operational parameters like the PM type, the slot opening and the flux densities inside the core branches will be pre-selected during the design procedure. The aim is to choose a configuration characterized by a low value of poles, in order to limit the inverter rated frequency and the core losses, hence increasing the motor efficiency. In Fig. 1 and Fig. 2 the adopted configuration and the motor quotes are displayed; Table I lists the design rated quantities and the material properties.

\section{(a)}
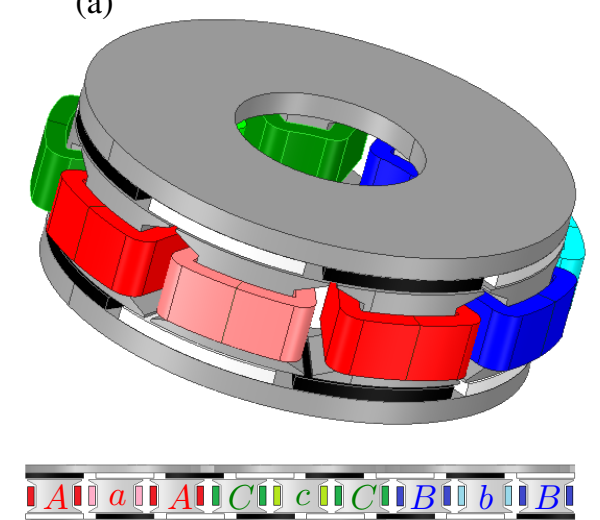

(b)

Fig. 1. (a) YASA double rotor single stator magnetic structure and (b) Phase coil sequence.
TABLE I

DESIGN DATA

\begin{tabular}{c|c|c|c}
\hline$T_{n}[N \cdot m]$ & $N_{n}[\mathrm{rpm}]$ & $P_{n}[\mathrm{~kW}]$ & $T_{w_{\text {out }}}-T_{w_{\text {in }}\left[{ }^{\circ} \mathrm{C}\right]}$ \\
\hline 700 & 900 & 66 & $70-60$ \\
\hline \multicolumn{2}{c}{ Permanent magnet grade } & $\mathrm{N} 33 \mathrm{EH}$ \\
\hline \multicolumn{2}{c}{ Stator and rotor magnetic material } & Steel-1010;17\%-CoFe-alloy \\
\hline \multicolumn{2}{c}{ Enamel and potting conductivity $\left[\frac{\mathrm{W}}{\mathrm{m} \cdot{ }^{\circ} \mathrm{C}}\right]$} & $0.25 ; 3.2$ \\
\hline
\end{tabular}

The number of poles is 8 and, considering a motor with just one cycle $\left(N_{c}=1\right)$ according to (1), the number of stator teeth chosen will be $N_{t}=9$.

$$
N_{t}=N_{m} \pm N_{c}
$$

This allow to obtain the highest value of distribution factor $k_{d}$ thus increasing the winding factor $k_{w}$ [5]. The reason for choosing a YASA configuration is to limit the iron mass of the yoke which is greater compared to higher poles machines. In addiction, the motor volume reduction increases the torque density [6].

\section{Geometry and Sizing Equations}

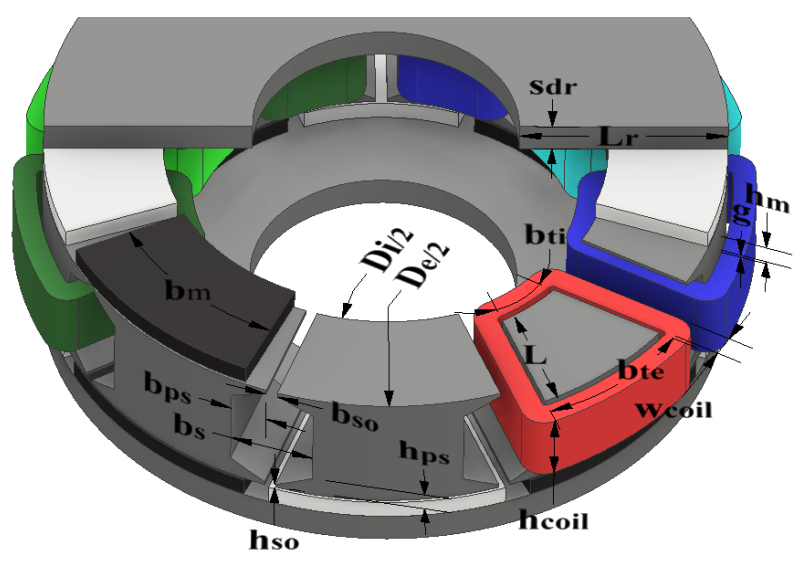

Fig. 2. Motor 3D geometrical structure with main quotes.

As well as in many papers the pre-design procedure starts from the sizing equations. Being an SPM motor the electromagnetic torque is the only significant contribute to the total torque generated and thus the rated design point of the motor will be set to the condition which maximize it. That is, when the rated current is in phase with the B.E.M.F generated by the PM flux. In such a condition, the electrical rated power of the machine is equal to three times the product of the rated phase B.E.M.F and the rated phase current.

$$
P_{n}=3 \cdot E_{p h_{n}} \cdot I_{p h_{n}}=T_{n} \cdot \frac{2 \cdot \omega_{e_{n}}}{N_{m}}
$$

Throughout (2) the value of the rated torque can be obtained:

$$
T_{n}=3 \cdot \frac{N_{m}}{2 \cdot \omega_{e_{n}}} \cdot E_{p h_{n}} \cdot I_{p h_{n}}
$$

the quantity $\frac{N_{m}}{2}$ corresponds to the number of pole pairs of the machine and $\omega_{e_{n}}$ is the rated electrical angular frequency. The rated line to neutral value of the B.E.M.F is:

$$
E_{p h_{n}}=\frac{k_{w} \cdot \omega_{e_{n}}}{a} \cdot N_{c} \cdot N_{c_{p h}} \cdot N_{t c} \cdot \frac{\Phi_{1}}{\sqrt{2}}
$$


where $a$ is the number of parallel paths, $N_{c}, N_{c_{p h}}, N_{t c}$ match up with the number of cycles, coils per phase and coil turns while $k_{w}$ is the winding factor of the $1^{\text {st }}$ harmonic B.E.M.F. Because of the single layer configuration, the winding factor, product of the layer displacement factor and the distribution factor, coincides with the distribution factor only. According to [7] its expression corresponds to (5).

$$
k_{w}=\frac{\sin \left(N_{c_{p h}} \cdot \frac{\alpha_{t}}{2}\right)}{N_{c_{p h}} \cdot \sin \left(\frac{\alpha_{t}}{2}\right)}
$$

Inside (4), the flux $\Phi_{1}$ is the pole flux corresponding to the fundamental component of the air gap flux density distribution calculated by (6).

$$
B_{1}=\frac{4}{\pi} \cdot B_{g} \cdot \sin \left(\alpha_{m} \cdot \frac{\pi}{2}\right)
$$

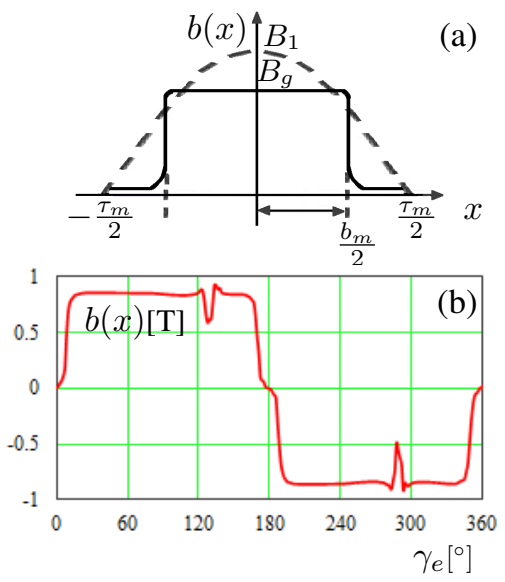

Fig. 3. (a) Ideal and (b) Real pole air gap flux density profile on the average radial position.

$\Phi_{g}$ is linked to the PM flux $\Phi_{m}$ by the equivalent circuit of Fig. 4. That is representative of a single air gap circuit, wherein the PM is aligned to the stator tooth. $\Theta_{m}, \Theta_{l}$ and $\Theta_{g}$ are respectively the internal PM, the leakage and the equivalent air gap reluctances.

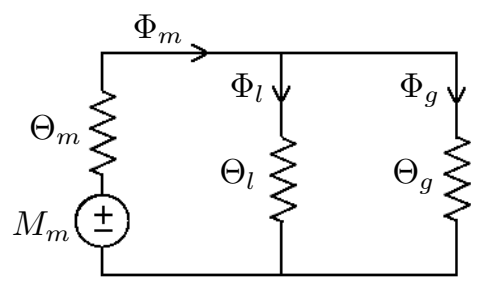

Fig. 4. PM-Tooth equivalent circuit, when PM and tooth are perfectly aligned.

$\Phi_{g}=\Phi_{m} \cdot \frac{\Theta_{l}}{\Theta_{l}+\Theta_{g}}=\Phi_{m} \cdot \eta_{M g}=\frac{M_{m}}{\Theta_{m}+\Theta_{g} \cdot \eta_{M g}} \cdot \eta_{M g}$

$\Phi_{g}$, difference between the PM flux and the leakage flux is the product of the flux produced by the magnet and $\eta_{M g}$, the working permanent magnet efficiency factor.
Choosing the flux density levels inside the core branches, according to Table II, leads to calculate the design rotor and stator geometrical parameters. Their values have been selected taking into account the effect at loaded conditions, where the influence of the winding currents locally increases the flux density into the magnetic circuit branches by a certain factor.

TABLE II

FLux Densities AT No LOAD

\begin{tabular}{cccc}
\hline$B_{r}[T]$ & $B_{t}[T]$ & $B_{d r}[T]$ & $B_{p s}[T]$ \\
\hline 1.1 & 1.65 & 2.2 & 1.4 \\
\hline
\end{tabular}

Approximately the flux crossing a tooth can be considered equal to the flux crossing a machine pole and according to it, the stator tooth area is the follows:

$$
A_{t}=\frac{\Phi_{t}}{B_{t}} \simeq \frac{\Phi_{g}}{B_{t}}
$$

considering $A_{t}$ shaped as trapezoidal, the slot peripheral size is written as a function of known geometrical variables:

$$
b_{s}=\frac{\pi \cdot D_{e} \cdot\left(1+\rho_{D}\right)}{2 \cdot N_{t}}-\frac{2 \cdot A_{t}}{k_{s t} \cdot D_{e} \cdot\left(1-\rho_{D}\right)}
$$

where $k_{s t}$ is the stator core stacking factor and $\frac{D_{e}}{2} \cdot\left(1+\rho_{D}\right)$ is the average diameter. In the same way the stator pole shoe height and rotor disks thickness will be:

$$
\begin{gathered}
h_{p s}=\frac{B_{g} \cdot b_{p s}}{B_{p s}} \\
s_{d r}=\frac{B_{m} \cdot A_{m}}{B_{d r} \cdot 2 \cdot L_{r}}
\end{gathered}
$$

with $B_{p s}, B_{m}$ the flux densities inside the body of the pole shoe and permanent magnets respectively and $B_{g}$ the maximum value of the air gap flux density profile shown in Fig. 3a, whose expression can be stated as:

$$
B_{g}=\frac{\Phi_{m}}{A_{m}} \cdot \eta_{M g}=B_{r} \cdot \frac{\eta_{M g}}{1+\frac{g}{h_{m} \cdot \mu_{p u}^{-1}} \cdot \eta_{M g}}
$$

$B_{r}$ is the residual PM flux density, $g$ and $h_{m}$ are the air gap and magnets heights.

Substituting (4) inside (3), the expression of the the RMS single coil M.M.F is obtained:

$$
M_{\text {coil }_{n}}=\frac{T_{n}}{\frac{k_{w}}{k_{c}} \cdot \frac{\left(N_{m}+1\right)}{4 \cdot \sqrt{2} \cdot a} \cdot D_{e}^{2} \cdot\left(1-\rho_{D}^{2}\right) \cdot B_{1}}
$$

its expression, product of $N_{t c}$ and $I_{p h_{n}}$ gives the magneto motive force necessary to get the rated torque according to the magnetic and geometrical constraints. From (6) the quantity $B_{1}$ multiplied by the pole area gives the flux $\phi_{1}$. Finally the flux crossing area has been reduced by the Carter's factor:

$$
k_{c}=\frac{1}{1-\frac{b_{s o} \cdot \sigma}{\tau_{c}}}
$$

$\sigma=\frac{2}{\pi} \cdot\left\{\arctan \left[\frac{b_{\text {so }}}{2 \cdot\left(g+\frac{h_{m}}{\mu_{r_{p u}}}\right)}\right]-\frac{g+\frac{h_{m}}{\mu_{r_{p u}}}}{b_{\text {so }}} \cdot \ln \left[1+\left(\frac{b_{s o}}{2 \cdot\left(g+\frac{h_{m}}{\mu_{r_{p u}}}\right)}\right)^{2}\right]\right\}$ 
to take into account the effect of the slot opening over the air gap flux density profile. Inside Table III some of the initial main design data have been included.

TABLE III

INITIAL DATA

\begin{tabular}{c|c|c|c|c|c}
\hline$D_{e}[\mathrm{~mm}]$ & 350 & $w_{c h}[\mathrm{~mm}]$ & 2 & $h_{m}[\mathrm{~mm}]$ & 10 \\
\hline$\rho_{D}$ & 0.65 & $w_{g}[\mathrm{~mm}]$ & 1 & $v_{w}\left[\frac{\mathrm{m}}{\mathrm{s}}\right]$ & 0.25 \\
\hline$L[\mathrm{~mm}]$ & 61.3 & $w_{e n}[\mathrm{~mm}]$ & 0.1 & $k_{s t} ; \alpha_{c u} ; \alpha_{m}$ & $0.95 ; 0.8 ; 0.8$ \\
\hline$L_{r}[\mathrm{~mm}]$ & 107 & $w_{\text {pot }}[\mathrm{mm}]$ & 2.5 & $\mu_{r_{p u}} ; \eta_{M g}$ & $1.04 ; 0.9$ \\
\hline$b_{s o}[\mathrm{~mm}]$ & 6 & $h_{s o}[\mathrm{~mm}]$ & 2 & $a ; N_{c} ; N_{c_{p h}}$ & $1 ; 1 ; 3$ \\
\hline$d_{c h}[\mathrm{~mm}]$ & 3 & $g[\mathrm{~mm}]$ & 1.5 & $N_{m} ; N_{t}$ & $8 ; 9$ \\
\hline
\end{tabular}

\section{THERMAL ASPECTS}

In the following section the calculation of the motor sizes is related with the thermal model in order to satisfy the temperature constraints as well. The cooling layout chosen is shown into Fig. 5. Each single coil wrapped around a stator tooth is inserted inside a cooling circuit and connected in parallel with the other coil cooling circuits. Each coil cooling circuit, whose sizes are $d_{c h}$ and $h_{\text {coil }}$, is wound all around the whole coil. The water flow removes the heat touching directly the potting that lies on to the lateral coil surface.

In first approximation the source of loss considered is the joule loss inside the winding only. Indeed, in this case, it is reasonable to assume that the joule loss is the main quantity responsible for the heat flux generated inside the machine due to the high current density.

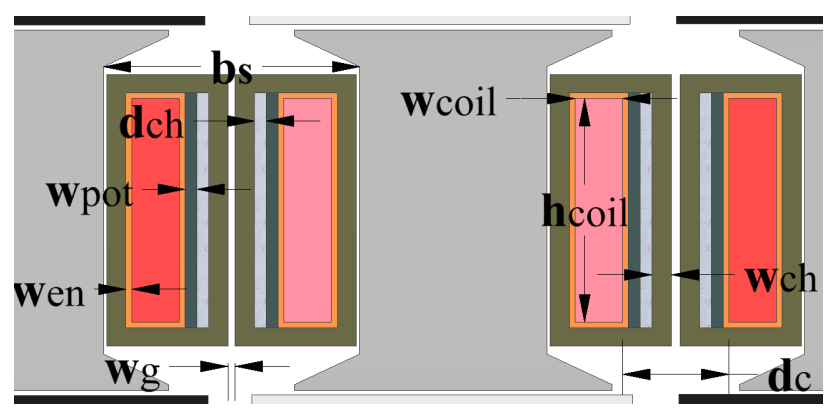

Fig. 5. Coil cooling layout front view.

The low number of poles implies a small amount of additional and iron losses during rated conditions.

$$
P_{c u}=\rho_{c u} \cdot S^{2} \cdot \alpha_{c u} \cdot h_{c o i l} \cdot w_{c o i l} \cdot l_{\text {turn }}
$$

Equation (15) expresses the DC loss inside a single coil, $S$ is the surface current density, $\rho_{c u}, \alpha_{c u}$ are the resistivity and the copper filling factor whereas $l_{\text {turn }}, h_{\text {coil }}, w_{\text {coil }}$ are the average coil turn length, height and width.

The thermal heat is supposed exchanged from the copper towards the water flow inside the cooling channel which fit into the slot. No heat exchange, instead is considered throughout the upper and lower coil surfaces and between the internal lateral coil surface and the stator tooth body. The power dissipated by a single coil surface towards the cooling channel is:

$$
P_{w}=K_{t h} \cdot l_{\text {cool }} \cdot h_{\text {coil }} \cdot \theta
$$

the quantity $l_{\text {cool }} \cdot h_{\text {coil }}$ is the lateral and external thermal exchange area of a single coil, $\theta$ is the temperature difference between the copper and the inlet water temperature and $K_{t h}$ is the global thermal exchange coefficient. As concerns the copper temperature limit, an F class temperature insulation is considered.

The expression of the global thermal exchange coefficient can be retrieved by the equivalent circuit pictured in Fig 6.

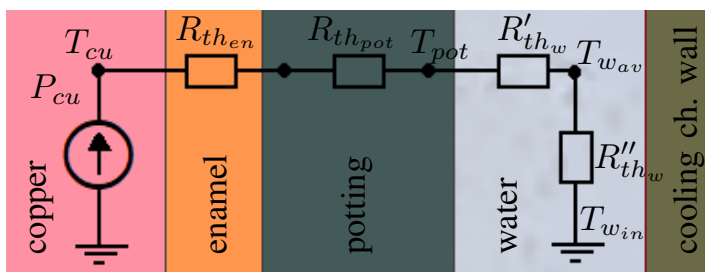

Fig. 6. Equivalent thermal circuit, representative of the coil cooling system during rated conditions.

The circuit is characterized by three thermal resistances: $R_{t h_{e n}}, R_{t h_{p o t}}$, and $R_{t h_{w}}$. The first two $R_{t h_{e n}}, R_{t h_{p o t}}$ are conductive resistances, representative of the conduction between the copper winding and its cover layers, enamel and potting. The third one $R_{t h_{w}}$, instead is a convective resistance, modeling the thermal exchange between the coil and the water inside the cooling channel. The two conductive thermal resistances, are:

$$
\begin{gathered}
R_{t_{\text {en }}} \simeq \frac{1}{\lambda_{\text {en }}} \cdot \frac{w_{\text {en }}}{\left(l_{\text {turn }}+4 \cdot w_{\text {coil }}\right) \cdot h_{\text {coil }}} \\
R_{t h_{\text {pot }}} \simeq \frac{1}{\lambda_{\text {pot }}} \cdot \frac{w_{\text {pot }}}{\left[l_{\text {turn }}+4 \cdot\left(w_{\text {coil }}+2 \cdot w_{\text {en }}\right)\right] \cdot h_{\text {coil }}}
\end{gathered}
$$

in the numerator the layers thickness and in the denominator the product of the thermal conductivity coefficients $\lambda_{e n}, \lambda_{p o t}$ (see Table I, values from Motorcad materials database) and the equivalent cooling surfaces respectively of the two insulation materials. Finally the thermal convective resistance of the fluid:

$$
R_{t h_{w}} \simeq R_{t h_{w}}^{\prime}+R_{t h_{w}}^{\prime \prime}=\frac{1}{\alpha_{w} \cdot h_{\text {coil }} \cdot l_{\text {cool }}}+\frac{1}{2 \cdot c_{w} \cdot \gamma_{w} \cdot Q}
$$

sum of two contributes: the equivalent convective resistance of the water/channel system and the energy absorbed by the water cooling channel flow $Q$. Quantities $c_{w}, \gamma_{w}$ are the specific heat and density of the water and $\alpha_{w}$ is the convective heat transfer coefficient of the channel.

Assuming turbulent flow inside it, the Dittus-Boelter's law can be used to model convection in line with procedure [8] using (20) and (21).

$$
\begin{gathered}
P_{w}=\alpha_{w} \cdot l_{\text {cool }} \cdot h_{c o i l} \cdot\left(T_{w_{\text {pot }}}-T_{w_{a v}}\right) \\
\alpha_{w}=\left[M_{o v}+K_{t w} \cdot\left(T_{w_{a v}}-T_{o v}\right)\right] \cdot d_{w}^{-0.2} \cdot v_{w}^{0.8} \\
P_{w}=c_{w} \cdot \gamma_{w} \cdot Q \cdot\left(T_{w_{o u t}}-T_{w_{i n}}\right) \simeq 2 \cdot c_{w} \cdot \gamma_{w} \cdot Q \cdot\left(T_{w_{a v}}-T_{w_{i n}}\right)
\end{gathered}
$$

$v_{w}$ is the average water speed into the cooling channel whilst $d_{w}$ is the cooling channel equivalent diameter that is the 
ratio between four times its area and twice its perimeter. The constants inside (20) are: $M_{o v}=1810, K_{t w}=19.7$, $T_{\text {ov }}=25^{\circ} \mathrm{C}$.

Splitting up the temperature drops inside the thermal circuit, $\theta$ equals the product between the sum of the thermal resistances and the total power delivered throughout the cooling surfaces:

$$
\begin{aligned}
\theta & =\left(T_{c u}-T_{p o t}\right)+\left(T_{p o t}-T_{w_{a v}}\right)+\left(T_{w_{a v}}-T_{w_{i n}}\right) \\
& =\left(R_{t h_{e n}}+R_{t h_{p o t}}+R_{t h_{w}}\right) \cdot P_{w}=R_{t h} \cdot P_{w}
\end{aligned}
$$

inside (22) the first temperature drop is in between the copper temperature and its covering surfaces, the enamel and potting layer; the second one is in between the wet potting layer surface touched by the water into the channel and its average temperature; the third one is the temperature drop in between the average water temperature and the inlet water temperature.

Afterwards looking at (16) and (22) by similarity the expression of the global thermal exchange coefficient is deduced.

$$
\begin{gathered}
K_{t h}=\frac{1}{\frac{l_{c o o l}}{d_{c h} \cdot 2 \cdot c_{w} \cdot \gamma_{w} \cdot v_{w}}+\frac{1}{\alpha_{w}}+\frac{w_{e n}}{\lambda_{e n}} \cdot \rho_{e n}+\frac{w_{p o t}}{\lambda_{p o t}} \cdot \rho_{p o t}} \text { (23) } \\
\rho_{\text {en }}=\frac{l_{\text {cool }}}{\left[l_{t u r n}+4 \cdot w_{\text {coil }}\right]} \quad \rho_{\text {pot }}=\frac{l_{\text {cool }}}{\left[l_{\text {turn }}+4 \cdot\left(w_{\text {coil }}+2 \cdot w_{\text {en }}\right)\right]}
\end{gathered}
$$

the therms $\rho_{\text {en }}, \rho_{\text {pot }}$ are the ratios of the external cooling perimeter $l_{\text {cool }}$ and the internal cooling perimeter of the winding cover layers which takes into account the reduction of thermal exchange perimeter of the most inner surfaces. The global thermal exchange coefficient is function of the coil height as much as the coefficient $\alpha_{w}$ is, and that is because of the equivalent cooling channel diameter which is function of $h_{\text {coil }}$ too.

Table IV shows the p.u resistance values with the external cooling surface as base unit $\left(h_{\text {coil }} \cdot l_{\text {cool }}\right)$, with an $h_{\text {coil }}=38$ $m m$.

$$
\begin{gathered}
\text { TABLE IV } \\
\text { P.U. Thermal RESistanCES }\left[\frac{{ }^{\circ} \mathrm{C} \cdot \mathrm{m}^{2}}{W}\right], h_{\text {coil }}=38[\mathrm{~mm}] .
\end{gathered}
$$

\begin{tabular}{cccc}
\hline$\frac{l_{\text {cool }}}{d_{c h} \cdot 2 \cdot c_{w} \cdot \gamma_{w} \cdot v_{w}}$ & $\alpha_{w}^{-1}$ & $\frac{w_{e n}}{\lambda_{e n}} \cdot \rho_{\text {en }}$ & $\frac{w_{p o t}}{\lambda_{p o t}} \cdot \rho_{\text {pot }}$ \\
\hline $5.64 \times 10^{-5}$ & $4.13 \times 10^{-4}$ & $4.25 \times 10^{-4}$ & $8.28 \times 10^{-4}$ \\
\hline
\end{tabular}

For what concern the water flow thermal resistance its value can be considered negligible compared to the other ones.

Equating (15) and (16) the motor surface current density can be expressed as a function of the coil height:

$$
S\left(h_{c o i l}\right)=\sqrt{\frac{K_{t h}\left(h_{c o i l}\right) \cdot l_{c o o l} \cdot \theta}{\rho_{c u} \cdot \alpha_{c u} \cdot w_{c o i l} \cdot l_{t u r n}}}
$$

equation (13) together with (24) are representative of the electromagnetic and thermal constraints. Thus finding the roots of (25) means finding the coil height value which satisfies them both, providing the requested rated torque and allowing to dissipate the DC losses generated by the machine coils for the considered design working point.

$$
\Delta\left(h_{\text {coil }}\right) \cdot h_{\text {coil }}-M_{\text {coil }}=0
$$

The quantity $\Delta\left(h_{\text {coil }}\right)$ is the linear current density over the coil axial length, function of the coil height as well as the surface current density. Its expression is stated in (26).

$$
\Delta\left(h_{\text {coil }}\right)=\alpha_{c u} \cdot S\left(h_{\text {coil }}\right) \cdot w_{\text {coil }}
$$

Fig. 7a and Fig. 7b show respectively the surface and linear current densities as a function of the variable coil height together with the coil height value which satisfies the rated thermal and electromagnetic constraints of (13) and (24).
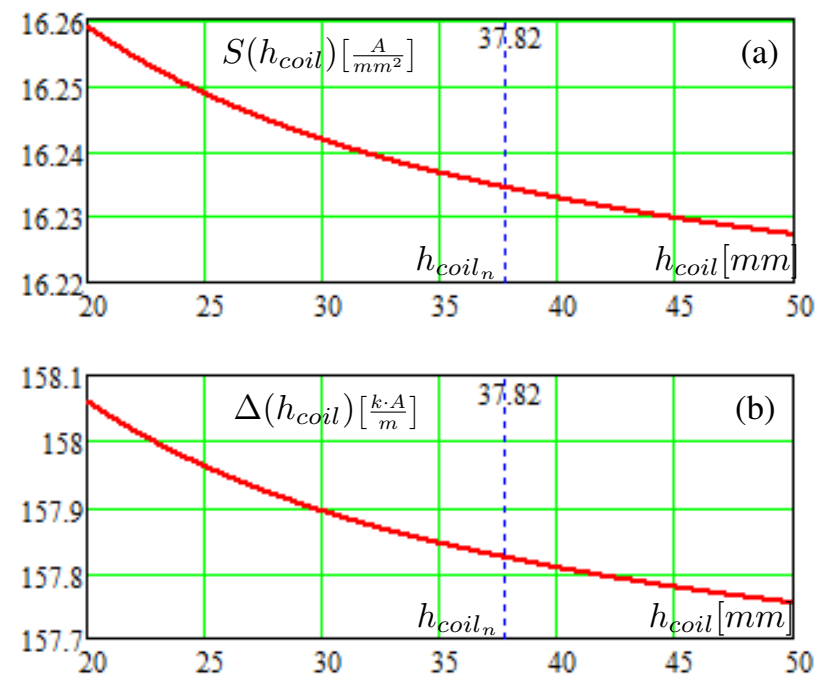

Fig. 7. Current densities as a function of the coil height: (a) surface (b) linear.

\section{WINDING DATA}

In order to calculate the motor winding data, the ratio between the B.E.M.F and the supply voltage identified as a key design parameter has to be evaluated. Inside Fig. 8 the torque-speed characteristic of the motor during peak and rated working conditions is shown.

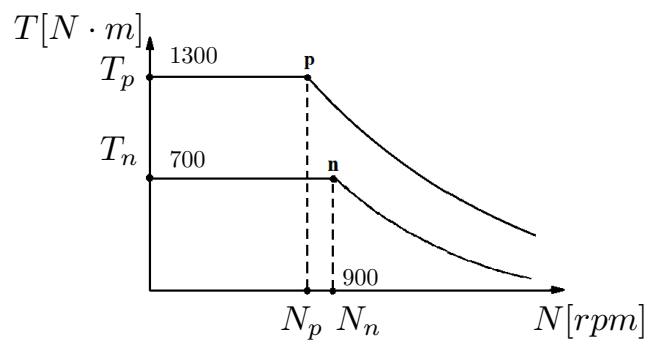

Fig. 8. Torque-speed characteristic during peak and rated conditions.

If the points $\mathrm{p}$ and $\mathrm{n}$ of Fig. 8 are considered as limit points in MTPA operation and saturation is neglected, the following proportionalities can be written:

$$
\frac{T_{p}}{T_{n}}=\frac{I_{p}}{I_{n}} ; \frac{N_{p}}{N_{n}}=\frac{E_{p}}{E_{n}} .
$$


Looking at the phasor diagram of the motor shown in Fig. 9, the design parameter $\rho_{E V}$ is defined:

$\rho_{E V}=\frac{E_{p h}}{V_{p h_{p}}}=\rho_{N} \cdot \rho_{E V_{n}}=\frac{\rho_{N}}{\sqrt{\left(\rho_{N}+\rho_{T} \cdot r\right)^{2}+\left(\rho_{T} \cdot \rho_{N} \cdot x\right)^{2}}}$

it is the ratio between the corner point B.E.M.F and the maximum supply voltage available at the inverter output terminals working in PWM, $V_{p h_{p}}=\frac{V_{d c}}{2 \cdot \sqrt{2}}$.

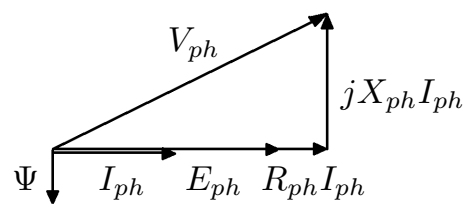

Fig. 9. Motor phasor diagram operating in MTPA.

Quantities $\rho_{N}, \rho_{T}$ are the speed and torque ratios and $r, x$ are the phase resistance and reactance in per unit, referred to the rated back electromotive force:

$$
\begin{gathered}
r=\frac{R_{p h} \cdot I_{p h_{n}}}{E_{p h_{n}}}=\frac{\rho_{c u} \cdot l_{t u r n} \cdot S_{n}}{\frac{k_{w}}{k_{c} \cdot a} \cdot \omega_{e_{n}} \cdot N_{c_{p h}} \cdot \frac{\Phi_{1}}{\sqrt{2}}} \\
x=\frac{X_{p h} \cdot I_{p h_{n}}}{E_{p h_{n}}}=\frac{L_{1} \cdot M_{c o i l_{n}}}{\frac{k_{w}}{k_{c} \cdot a} \cdot N_{c_{p h}} \cdot \frac{\Phi_{1}}{\sqrt{2}}}
\end{gathered}
$$

in which $L_{1}$ is the phase inductance with one coil turn only and it can be obtained thorough FEM simulations or by using an analytical approach [7].

Considering the rated corner point, the values $\rho_{N}$ and $\rho_{T}$ equal one and consequently the rated voltage ratio can be retrieved from (31).

$$
\rho_{E V_{n}}=\frac{E_{p h_{n}}}{V_{p h_{p}}}=\frac{1}{\sqrt{(1+r)^{2}+x^{2}}}
$$

Once the ratio $\rho_{E V_{n}}$ is calculated, chosen the peak torque ratio $\rho_{T_{p}}=\frac{T_{p}}{T_{n}}$, the value of $\rho_{N}$ can be obtained from (28) according to (32).

$$
\rho_{N}=\rho_{T_{p}} \cdot \frac{x \cdot \sqrt{\frac{1}{\rho_{E V_{n}}^{2}}+\frac{1}{\rho_{E V_{n}}^{2} \cdot \rho_{T_{p}}^{2} \cdot x^{2}}-r^{2} \cdot \rho_{T_{p}}^{2}}-r}{1+\rho_{T_{p}}^{2} \cdot x^{2}}
$$

Thanks to the design ratio $\rho_{E V_{n}}$ the evaluation of the coil turns number $N_{t c}$ is now possible:

$$
N_{t c}=\text { floor }\left(N_{t c_{t h}}\right)=\text { floor }\left(\frac{\rho_{E V_{n}} \cdot m_{a} \cdot V_{p h_{p}}}{\frac{k_{w}}{k_{c} \cdot a} \cdot \omega_{e_{n}} \cdot N_{c_{p h}} \cdot \frac{\Phi_{1}}{\sqrt{2}}}\right)
$$

the quantity $m_{a}$ is the inverter amplitude modulation ratio which has been set to 0.85 to keep a margin from the maximum voltage value.

The current density which flows inside the winding is high, the adoption of a single cable, would mean having a wire diameter which is greater than $4 \mathrm{~mm}$, smaller wire connected in parallel is not recommendable due to currents loop that may flow inside it. Hence it is preferable to use a copper strip wire, which has a higher filling factor also.
Adopting the starting, simplifying hypothesis that the strip has a perfect rectangular shape its area is:

$$
A_{p l_{t h}}=\frac{M_{c o i l}}{S \cdot N_{t c}}
$$

and the strip sizes accordingly should be as follows:

$$
\begin{gathered}
s_{p l_{t h}}=\frac{h_{c o i l}-\left(N_{t c}+1\right) \cdot 2 \cdot w_{e n}}{N_{t c}+1} \\
w_{p l_{t h}}=\frac{A_{p l_{t h}}}{s_{p l_{t h}}}
\end{gathered}
$$

bearing in mind the strip manufacturing constraint, the closest normalized strip is selected on the data-sheet. The theoretical and actual strip quantities calculated are summarized inside Table V.

TABLE V

WINDING STRIP DATA

\begin{tabular}{l|c|c|c|c}
\cline { 2 - 5 } & $s_{p l}[\mathrm{~mm}]$ & $w_{p l}[\mathrm{~mm}]$ & $A_{p l}\left[\mathrm{~mm}^{2}\right]$ & $S_{n}\left[\frac{\mathrm{A}}{\mathrm{mm}^{2}}\right]$ \\
\hline Theoretical & 1.38 & 11.62 & 15.99 & 16.23 \\
\hline Actual & 1.4 & 12 & 16.59 & 15.64 \\
\hline
\end{tabular}

The rated current density is slightly lower keeping a safety margin from the theoretical condition. The calculation of the thermal resistances and losses considering the normalized strip data during rated conditions reveals a copper temperature $T_{c u_{n}}=152^{\circ} \mathrm{C}$ in accordance with the insulation thermal limits.

\section{HEAT SHOCK}

In this section the heat shock condition is considered. Due to its rapidity the thermal phenomenon is considered to be adiabatic. All the heat developed by the winding is accumulated inside the copper volume; thus the following expression is written:

$$
\rho_{c u} \cdot S_{p}^{2} \cdot V_{c u}=c_{c u} \cdot \gamma_{c u} \cdot V_{c u} \cdot \frac{d \theta}{d t}
$$

the first term is the joule loss generated inside the winding, $S_{p}$ is the peak current density into the copper strip, $c_{c u}, \gamma_{c u}$ are the copper specific heat and density respectively and $V_{c u}$ is the copper volume. The copper resistivity $\rho_{c u}$, function of the copper strip temperature can be written as:

$$
\rho_{c u}(T)=\rho_{c u_{o}} \cdot\left[1+\alpha_{T_{c u}} \cdot\left(T-T_{a}\right)\right]
$$

in which $\rho_{c u}, \alpha_{T_{c u}}$ are the resistivity and the copper thermal coefficient and $T_{a}$ is the ambient temperature.

Using (38) with the resistivity of the copper at the rated temperature $T_{c u_{n}}$ calculated in the previous section and then substituting into (37) gives rise to a first order differential equation.

$$
c_{c u} \cdot \gamma_{c u} \cdot \frac{d \theta}{d t}-\rho_{c u_{n}} \cdot S_{p}^{2} \cdot \alpha_{T_{c u}} \cdot \theta=\rho_{c u_{n}} \cdot S_{p}^{2}
$$


Its solution describes the transient temperature rise profile of the copper strip wire working at peak current density, within the rated temperature as initial condition.

$$
\begin{gathered}
\theta=\left(\theta_{n}+\frac{1}{\alpha_{T_{c u}}}\right) \cdot e^{\lambda \cdot t}-\frac{1}{\alpha_{T_{c u}}} \\
\lambda=\frac{\rho_{c u_{n}} \cdot S_{n}^{2} \cdot \alpha_{T_{c u}}}{c_{c u} \cdot \gamma_{c u}} \cdot\left(\frac{S_{p}}{S_{n}}\right)^{2}
\end{gathered}
$$

Throughout (40) the maximum time allowed for the overload working condition, in accordance with the maximum temperature limits of the materials can be calculated by:

$$
\Delta t=\frac{1}{\lambda} \cdot \ln \frac{\theta_{M}+\frac{1}{\alpha_{T_{c u}}}}{\theta_{n}+\frac{1}{\alpha_{T_{c u}}}}
$$

whose initial conditions and results are stated into Table VI.

TABLE VI

OVERLOAD INITIAL DATA AND RESULTS

\begin{tabular}{cccc}
\hline$\theta_{n}\left[{ }^{\circ} C\right]$ & $\theta_{M}\left[{ }^{\circ} C\right]$ & $S_{p} / S_{n}$ & $\Delta t[s]$ \\
\hline 91.93 & 140 & 2.5 & 3.68 \\
\hline
\end{tabular}

\section{MAIN RESULTS}

Conclusively in this section some of the results retrieved from the previous analytical approach and the FEM validation will be shown. Fig. $10 \mathrm{~b}$ and $10 \mathrm{a}$ shows respectively the B.E.M.F and the torque retrieved using 3D FEM analyses.
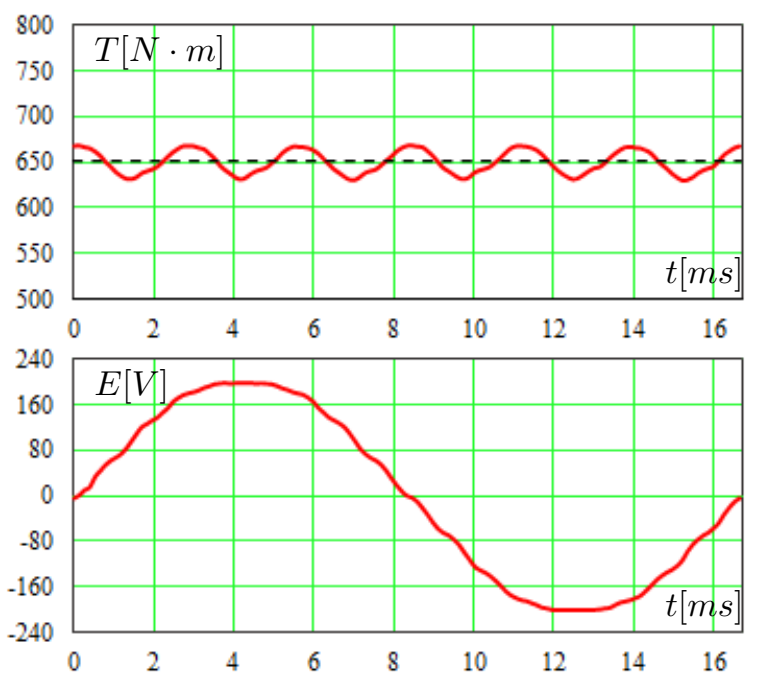

Fig. 10. (a) Torque and (b) No load phase to phase B.E.M.F during rated conditions.

The average torque with respect to the design rated torque differs by about $7.1 \%$, mainly due to uncertainties regarding pre-design data and local saturation. Table VII shows some results of the analytical procedure and the total masses.

TABLE VII

Calculation Results

\begin{tabular}{c|c|c|c|c|c}
\hline$b_{s}[\mathrm{~mm}]$ & 44.3 & $s_{d r}[\mathrm{~mm}]$ & 13 & $M_{c u} ; M_{P M}[\mathrm{~kg}]$ & $9 ; 7.5$ \\
\hline$h_{c o i l_{n}}[\mathrm{~mm}]$ & 37.8 & $P_{c u_{n}}[\mathrm{~W}]$ & 708 & $M_{f e}[\mathrm{~kg}]$ & 35 \\
\hline$h_{p s}[\mathrm{~mm}]$ & 12 & $N_{t c}$ & 23 & $M_{p o t}[\mathrm{~kg}]$ & 1 \\
\hline
\end{tabular}

\section{CONCLUSION}

The presented paper carries out a simple analytical predesign procedure in order to design an AFPM YASA inwheel motor with high torque density. The procedure started setting up initial geometrical constraints and the desired flux densities into the iron branches, beneficial to get the main core sizes for the electromagnetic circuit. Using the classical approach, based on the motor sizing equations, the utilization required to get the rated torque has been retrieved. By the use of the equivalent thermal and electromagnetic circuits the geometrical design parameter $h_{\text {coil }}$ closely related to the stator core tooth axial coordinate has been bounded, both to the electromagnetic and thermal equations. Its evaluation has allowed to satisfy the defined constraints and finally obtain the winding data. 3D FEM simulations have shown a torque error estimation around $7 \%$.

\section{REFERENCES}

[1] D. Luque, E. Ruppert, N. Bianchi, and M. Castiello, "Analysis of a threephase in-wheel electric motor," in 2009 44th International Universities Power Engineering Conference (UPEC), Sep. 2009, pp. 1-5.

[2] P. Virtič, M. Vražić, and G. Papa, "Design of an axial flux permanent magnet synchronous machine using analytical method and evolutionary optimization," IEEE Transactions on Energy Conversion, vol. 31, no. 1, pp. 150-158, March 2016.

[3] Y. Park, M. Koo, S. Jang, J. Choi, and D. You, "Performance evaluation of radial- and axial-flux pm wind power generators with mechanical energy storage system," IEEE Transactions on Energy Conversion, vol. 30, no. 1, pp. 237-245, March 2015.

[4] A. Di Gerlando, G. M. Foglia, M. F. Iacchetti, and R. Perini, "Sizing comparison of axial flux pm motors, for automotive application," in 2014 Intern. Conf. on Electrical Machines (ICEM), Sep. 2014, pp. 1409-1414.

[5] A. Di Gerlando, G. Foglia, M. F. Iacchetti, and R. Perini, "Axial flux pm machines with concentrated armature windings: Design analysis and test validation of wind energy generators," IEEE Transactions on Industrial Electronics, vol. 58, no. 9, pp. 3795-3805, Sep. 2011.

[6] B. Zhang, T. Seidler, R. Dierken, and M. Doppelbauer, "Development of a yokeless and segmented armature axial flux machine," IEEE Transactions on Industrial Electronics, vol. 63, no. 4, pp. 2062-2071, April 2016.

[7] A. Di Gerlando, G. M. Foglia, R. Perini, and M. Ubaldini, "Design and operation aspects of field regulated pm synchronous machines with concentrated armature windings," in IEEE Intern. Conf. on Electric Machines and Drives, 2005., May 2005, pp. 1165-1172.

[8] A. Di Gerlando, G. M. Foglia, M. F. Iacchetti, and R. Perini, "Thermal modeling for the design and check of an axial flux pm motor," in 2014 Intern. Conf. on Electrical Machines (ICEM), Sep. 2014, pp. 1441-1447.

\section{BiogRAPHIES}

Antonino Di Gerlando received his MS degree in electrical engineering from the Politecnico di Milano, Italy, in 1981. Currently, he is a Full Professor at the Department of Energy at Politecnico di Milano. Fields of interest: design and modeling of electrical machines, converters and drive systems. He is a senior member of IEEE and a member of the Italian Association of the Electric and Electronic Engineers (AEI).

Giovanni Maria Foglia received his MS degree and the $\mathrm{PhD}$ in electrical engineering at Politecnico di Milano, Milano, Italy, in 1997 and 2000. Currently, he is an Associate Professor at the Department of Energy at Politecnico di Milano, and his main field of interest is the analysis and design of PM electrical machines.

Claudio Ricca received his MS degree in electrical engineering in 2017 at Politecnico di Milano, Italy. Currently, he is a PhD student at Politecnico di Milano. His interests are in design and modeling of electrical machines and drives. 